\title{
EVALUACIÓN DEL IMPACTO DE LA SALUD DE LAS MUJERES EN EL ÁREA DE CURRIDABAT (TIRRASES), SAN JOSÉ, COSTA RICA
}

\author{
Licda. Delfina Charlton L.
}

Profesora de Enfermería de La Universidad de Costa Rica San Pedro, Montes De Oca

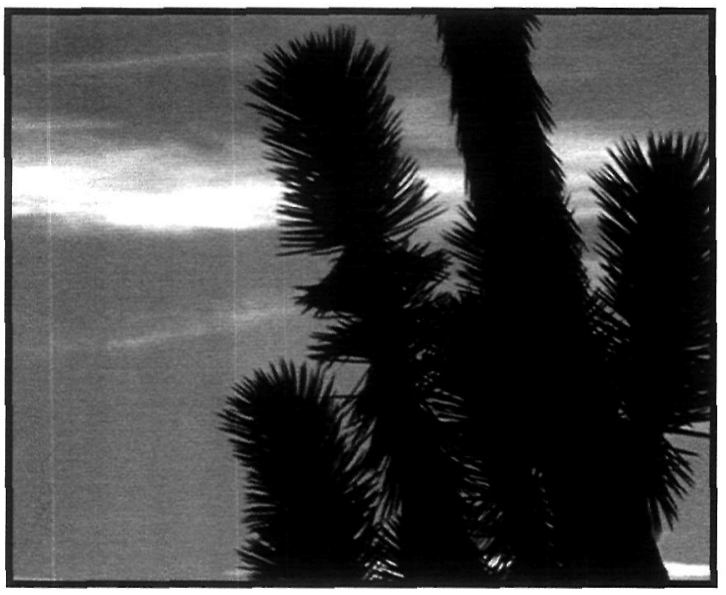

EVALUATION OF THE IMPACT OF WOMEN ON HEALTH IN CURRIDABAT (TIRRASES), SAN JOSE, COSTA RICA

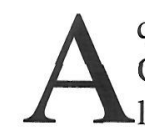
qualitative research was carried out in Curridabat (Tirrases, Costa Rica) by 10 leader women whose aim was helping their community to improve health conditions, achieve women empowerment, autonomy and adequate decision making, taking in consideration social, political and economical aspects.

Three group sessions dealt with health determining factors in the social, political and economical areas respectively.

Later, according to the objectives, a qualitative data analysis was performed and the results are presented in this work.

\section{RESUMEN}

$\mathrm{E}$ 1 presente artículo es una investigación con metodología cualitativa, llevada a cabo en el Cantón de Curridabat (Tirrases) por 10 mujeres líderes de esa comunidad, cuyo interés primordial es ayudar a su comunidad para mejorar las condiciones de salud de esa población, conocer las estrategias para manejar la salud, lograr el empoderamiento de las mujeres, su autonomía y adecuada toma de decisiones para su vida personal y familiar, tomando en cuenta las áreas social, política y económica del estudio.

Para el desarrollo y logro de los objetivos propuestos, se trabajó con tres sesiones de grupos focales en diferentes momentos; abarcando diversos aspectos. En la primera sesión se trabajó "los determinantes de la salud en el área social" donde se elaboró una guía de preguntas abiertas distribuidas en categorías de género, salud y prácticas cotidianas. En la segunda sesión se desarrolló la temática "los determinantes de la salud en el área política", con ello se abarcó la acción comunitaria y política. En la tercera sesión se desarrollaron "los determinantes de la salud en el área económica", tomando en cuenta los recursos financieros y acceso a los servicios de salud de esa población.

Posteriormente, de acuerdo a los objetivos del trabajo investigativo, se realizó un análisis del contenido de la información en forma cualitativa, luego se hace una triangulación de los datos obteniendo los resultados que a continuación se describen.

\section{LEXEMAS:}

Administradoras del hogar: se refiere a las mujeres amas de casa.

\section{EBAIS:}

Se refiere al Equipo Básico de Atención integral en salud que existe en las áreas de salud de Costa Rica. 


\section{DETERMINANTES DE LA SALUD:}

Son las situaciones de salud que forman parte de la cotidianidad del individuo, me refiero al modo de vida, estilo de vida de cada persona y sus prácticas de salud, así como los problemas de salud a los que se enfrentan.

\section{AUTONOMÍA DE LAS MUJERES:}

Conjunto de procesos de poder. Es construir un conjunto de derechos que aseguren un tipo de libertad para las mujeres.

\section{INTRODUCCIÓN}

โ

a mujer es la clave en el proceso del desarrollo y en este momento es el sector más pobre entre los pobres. La eliminación de la discriminación, tanto social como cultural, política y económica, se ve reflejada en un alto servicio de salud sexual y salud reproductiva de calidad, que aportará beneficios en cuanto a: la mujer tiene autonomía, sobre todo en el programa de desarrollo humano. En este momento se carece a nivel mundial de esta autonomía y se deja sentir, aún más, en países en vías de desarrollo como lo es el caso de Latinoamérica. En un informe brindado por la Conferencia Mundial sobre Educación para todos en 1990, realizada en Tailandia, se refiere que 960 millones de adultos son analfabetos, de esa cantidad, dos terceras partes son mujeres; y que 130 millones de niños que no van a la escuela, el $70 \%$ son niñas. Es bien conocido que la mujer permanece en desigualdad de condiciones con respecto al hombre, sobre todo lo que concierne al desarrollo tanto social, económico, político y cultural. La participación de la mujer es escasa en estos ámbitos, ya que se le ha asignado el escenario doméstico, justificado por la visión enfocada a la función procreadora inherente a la femineidad, además a nivel de género el marginamiento que sufre la mujer es alto, en cuanto a la pobreza es la más perjudicada. Esta investigación permite detectar cuáles son los determinantes de la salud de la mujer que se caracterizan por su estabilidad económica, la visión de género y la triple jornada de trabajo (cuidado del hogar, desempeño reproductivo y trabajo), así como la desigualdad de oportunidades entre ellas mismas. Los resultados de esta investigación serán un aporte para el proyecto de atención de salud en los cantones de Curridabat, que tiene la Universidad de Costa Rica en convenio con la Caja Costarricense de Seguro Social.

\section{OBJETIVOS}

Generales: desarrollar un perfil de los determinantes de la salud de las mujeres de Tirrases, así como el desarrollo colectivo de alternativas dirigidas hacia la promoción de la salud de la familia, grupos comunitarios y comunidad.

\section{Específicos:}

- Describir los determinantes de salud y salud reproductiva desde la perspectiva de género en el marco económico, social y político.

- Conocer las prácticas de salud de esas mujeres, sus creencias y mitos que manejan en la cotidianidad.

- Realizar en colaboración con las mujeres de la comunidad, desde el área social, económica y política, la construcción colectiva de alternativas para el mejoramiento de su salud.

\section{SUJETOS DE ESTUDIOS}

Para la presente investigación se trabajó con diez mujeres de la comunidad de Tirrases, las cuáles son líderes comunales. No se contó con la participación de los varones, porque no acudieron a las reuniones, a pesar de haberles invitado. Las reuniones se llevaron a cabo en grupos focales en tres oportunidades diferentes. En el primer momento se desarrollaron los determinantes de la salud en la esfera social. En el segundo momento se tomó en cuenta los aspectos políticos. En el tercero momento se discutieron los determinantes de la salud en la esfera económica. Con estas tres actividades desarrolladas se concluyó la investigación y se estableció el compromiso de otra reunión para dar a conocer los resultados y la socialización de la información. El análisis de los datos se llevó a cabo en forma cualitativa, con el fin de conocer la opinión de las mujeres de Tirrases acerca de su estado de salud y los determinantes que emplean para mantenerse en buen estado de salud, tomando en cuenta las esferas social, económica y política. 
El desarrollo de la investigación tuvo una duración de seis meses.

Para ubicar al lector, se describe el lugar de la investigación:

Tirrases, Curridabat, San José, Costa Rica. una población urbano - marginal que cuenta con 15.000 habitantes; comprende tres sectores distribuidos en:

Tirrases $1-4.893$ habitantes -6 barrios

Tirrases $2-3.980$ habitantes -7 barrios

Tirrases $3-2.587$ habitantes -5 barrios

Las características más relevantes son: Viviendas de un solo aposento; problemas de pobreza; población inmigrante, indocumentada, que no asiste a los servicios de salud; principales problemas de salud: embarazo en adolescentes, prostitución, agresión física y sexual, abuso de drogas y precarismo.

Características de las mujeres del estudio: La población participante se ubica en el rango de edades comprendido entre los 31 y los 45 años. Edad reproductiva y de múltiples actividades en su cotidianidad. Todas las integrantes son costarricenses por lo que se observó el compromiso establecido con su comunidad y la lucha por su superación y bienestar. E1 90\% de las mujeres son administradoras del hogar y a pesar de sus múltiples ocupaciones en el hogar, se dedican a ayudar a otros (as) por el bienestar de su comunidad. En cuanto a su escolaridad, el nivel básico es el que predomina.

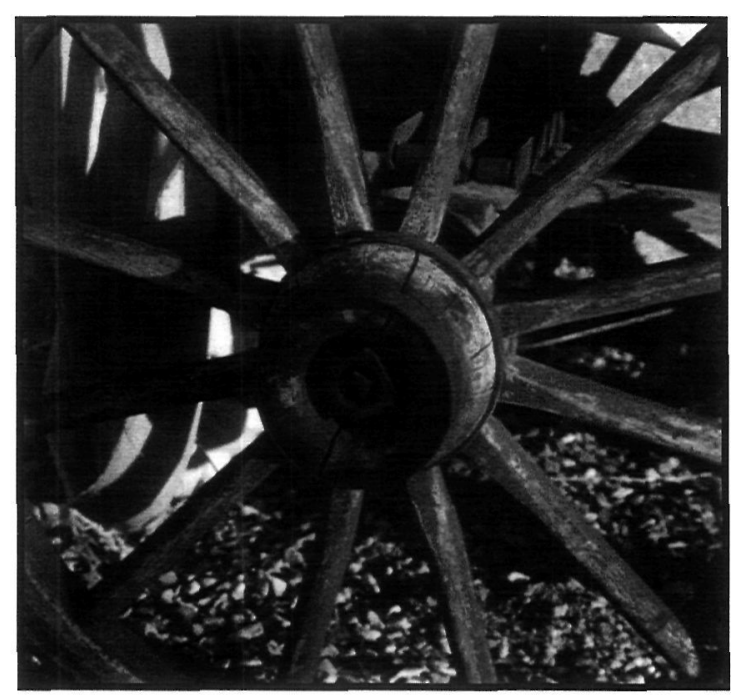

\section{RESULTADOS:}

En este apartado se analizan las voces de las mujeres de Tirrases, haciendo uso de nombres ficticios para asegurar su confidencialidad. Estos datos fueron categorizados en las siguientes áreas:

a) Determinantes de la salud en el área social abarcando género, salud y prácticas cotidianas.

b) Determinantes de la salud en el área económica, en este rubro se toman en cuenta los recursos financieros disponibles, emergencias en salud, acceso a los servicios de salud.

c) Determinantes de la salud en el área política, abarcando aspectos de acción comunitaria y política, con estos se cubre el objetivo $n^{\circ} 1$ de la investigación.

a) Los resultados obtenidos de la investigación, se evidencia en los siguientes logros en el Área Social:

Se observa un empoderamiento de estas mujeres en el cuidado de su salud y la preocupación por ayudar a otras para evitar enfermedades del aparato reproductor, asimismo se establece una buena organización para controlar la salud de la comunidad.

En cuanto a los determinantes de salud, ellas no lo mencionan como tal, sin embargo, se observa que analizan la situación de salud de su comunidad como parte de su problema de salud, lo cual las están afectando en diferentes circunstancias.

El grupo en general tiene una conciencia clara de que la salud sexual y reproductiva es alcanzar el nivel más elevado del bienestar físico y psicológico a través del goce sano responsable y seguro y el cuidado óptimo de su salud evitando riesgos de enfermar.

Otros resultados de la investigación que llaman la atención son los siguientes:

La mayoría de las mujeres de Tirrases emplean la recreación como una buena práctica de salud acompañada de estilos de vida favorables que contribuyan al beneficio de su salud sin descuidar la responsabilidad y la conciencia social.

Una de las participantes hace mención de que "la mujer no se puede enfermar, porque se detienen las actividad de la casa", sin embargo en la sociedad patriarcal este aspecto es subestimado por la 
invisibilidad que se le da al trabajo que realizan las mujeres en la esfera doméstica.

Las mujeres del grupo focal sienten la necesidad de buscar estrategias urgentes para mejorar la salud de la población, por lo tanto es necesario promover espacios para identificar las necesidades de salud existentes y buscar soluciones en conjunto a los problemas encontrados. También es urgente proporcionar información necesaria para tomar decisiones en la vida diaria que les permita proteger su salud, se evidencia que los servicios de salud no dan una respuesta adecuada a la problemática. Se observa la necesidad de educar a la población de Tirrases, para que se autocuiden y busquen atención oportuna en caso de enfermedad, asimismo que se organicen con otras mujeres para enfrentar solidariamente los problemas de salud para mantenerse saludables.

b) Los resultados que se obtuvieron en el área política son los que se mencionan a continuación:

Las mujeres del grupo focal están altamente motivadas para participar activamente en el voluntariado y contribuir al bienestar de la comunidad en general y al mejoramiento de las condiciones de salud en particular. Los vínculos establecidos con el EBAIS son bastante positivos y existe consenso entre las participantes en cuanto a la valoración del trabajo que ahí se realiza. La seriedad del personal de salud y los programas desarrollados han establecido un clima de confianza que redunda en una mayor participación y motivación de parte de estas líderes.

Los problemas del suministro de agua y de energía eléctrica se agravan por cuanto por habitar precarios, estas personas se conectan a los servicios de la mejor manera que pueden, al hacerlo crean problemas de suministros y no aparecen registrados como usuarios directos de los servicios.

c) En los determinantes económicos o área económica se pueden mencionar los siguientes resultados:

La atención de la enfermedad y del cuidado correspondiente a la mujer en la medida de que se pueda realzar por los medios con que cuenta la familia. Cuando esto no es así, se recurre a los espacios institucionales (Caja Costarricense de Seguro Social), el costo de estos servicios, repre- sentado por la cuota semanal o mensual. Así la atención de la salud es percibida como algo muy importante en las condiciones de vida de la familia. Para procurarse su atención, lo primero es conseguirse el acceso a los servicios públicos institucionalizados, tanto de enfermedad como de pensión. Para la prevención, la enfermedad y el cuidado cotidianos, se procura atenderlo dentro del hogar, en la medida de las posibilidades económicas y con la ayuda de familiares cercanos. La fragilidad económica, la distribución etaria de las participantes y las categorías ocupacionales de los jefes de familia, contribuyen a aumentar el peso de esa fragilidad y son fuente de gran tensión.

En efecto los bajos ingresos, el hecho de que los hijos se casen y dejen el hogar paulatinamente, contribuye a disminuir más los ingresos familiares y éstos tienen que ser distribuidos para atender todas las necesidades del hogar.

Objetivo Específico n²: Conocer las prácticas de salud de esas mujeres, sus creencias y mitos que manejan en la cotidianidad. Este objetivo se logró en las sesiones de grupo focal analizadas de la siguiente manera:

Las prácticas de salud de las mujeres de Tirrases está basada específicamente al mejoramiento de un estilo de vida saludable que les permite desenvolverse en un ambiente que favorezcan su salud.

Las mujeres de Tirrases emplean la recreación como una buena práctica de salud acompañada de estilos de vida favorables que contribuyan al beneficio de su salud sin descuidar la responsabilidad y la conciencia social. También se menciona la importancia de mantener un ambiente saludable y limpio en el hogar y la comunidad. Sin embargo no mencionan las buenas relaciones interpersonales, cordiales y satisfactorias en la familia, el vecindario y la comunidad, aspecto importante de reforzar en estas prácticas cotidianas. Implementar la promoción de la salud como una estrategia conjunta que permita fortalecer el acceso a la salud de toda la población.

En el análisis del mito mencionado por una de las participantes "si la mujer se enferma se cae la casa", al respecto se dice que la sociedad espera que las mujeres sean activas y autosuficientes en la 
esfera doméstica, en donde no tienen derecho a enfermarse, esta lucha cotidiana de atender su familia hace que seamos sensibles al deterioro de la salud. Los mitos mencionados por las mujeres de Tirrases son dados por una sociedad androcéntrica, distorsionados por una ideología que asume características específicas de discriminación hacia la mujer.

Objetivo Específico n³: Realizar en colaboración con las mujeres de la comunidad y los profesionales en salud, así como en las áreas económica, social y política, la construcción colectiva de alternativas para el mejoramiento de su salud.

La construcción colectiva de estrategias para el mejoramiento de la salud de la población de Tirrases, al respecto las mujeres manifiestan que: La necesidad de buscar estrategias urgentes para mejorar la salud de la población por lo tanto es necesario promover espacios para identificar las necesidades de salud existentes y buscar soluciones en conjunto a los problemas encontrados.

También refieren que es urgente proporcionar información necesaria para tomar decisiones en la vida diaria que les permita proteger su salud, se evidencia que los servicios de salud no dan una respuesta adecuada a la problemática de la comunidad.

Se observa la necesidad de educar a la población de Tirrases, para que se autocuiden y busquen atención oportuna en caso de enfermedad, asimismo que se organicen con otras mujeres para enfrentar solidariamente los problemas de salud para mantenerse saludables.

\section{DISCUSIÓN}

En resumen, estos objetivos se pueden analizar en forma integrada, tomando en cuenta las tres áreas, social, económica y política, de la salud de las mujeres.

La dimensión social de la salud fue abordada a partir de las condiciones de género y autoestima, la salud y las prácticas de salud. El análisis de las condiciones económicas abordó las dimensiones financieras y finalmente la dimensión política enfatizó los aspectos de participación política.
En cuanto a los aspectos de género y autoestima se determinó que las mujeres del grupo focal tienen internalizada su autonomía y adecuada toma de decisiones, y ésta se manifiesta en la importancia que las mismas conceden al brindar ayuda solidaria a otros con apoyo, amor y dedicación, para que todos juntos puedan construir, por medio de la igualdad, mejores condiciones de salud en esa comunidad. Esta percepción se relaciona directamente y refuerza la visión que estas mujeres poseen de su participación comunitaria.

En relación a las percepciones en salud, se reconoce que la salud es un factor fundamental del bienestar general, lo que hace que ellas asuman como responsabilidad el detectar los problemas que afectan las condiciones de salud y buscarles solución dentro del grupo familiar. Estas mujeres se perciben como responsables del bienestar total de las familias y dentro de ese bienestar, de la salud de sus miembros. Desde este punto de vista la mujer adopta comportamientos que le garantizan recursos para dar respuesta a las necesidades de salud.

En sus prácticas cotidianas, las mujeres manifiestan preocupación por la apropiación al mantenimiento de una buena salud, como lo son la higie-

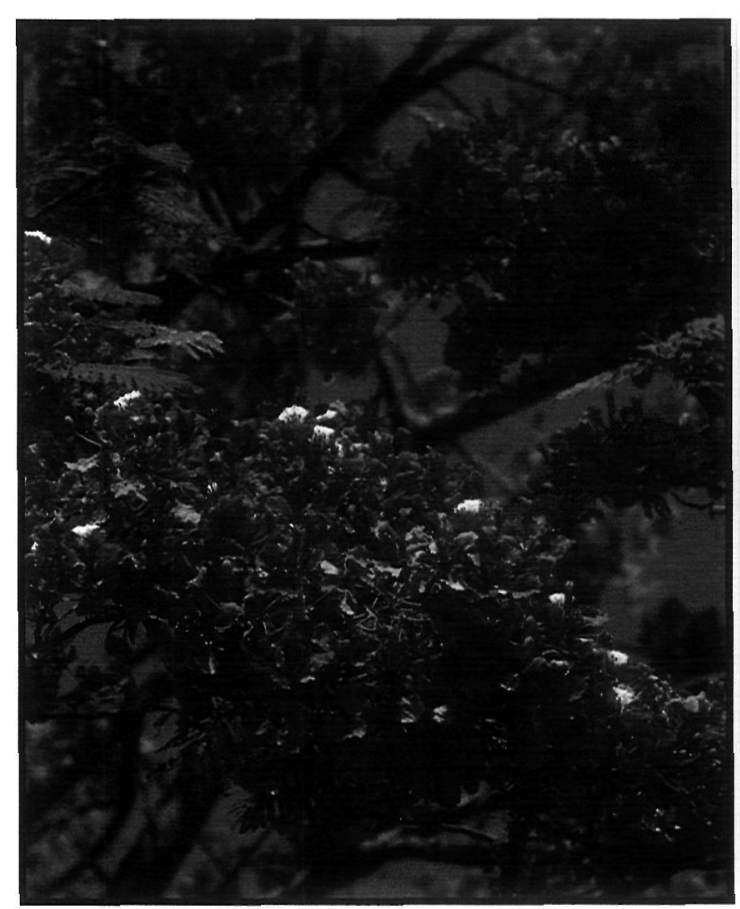


ne, el descanso adecuado, la alimentación y los cuidados necesarios para enferme-dades menores. En este respecto, estas mujeres desarrollan estrategias para garantizar estas condiciones que promueven una buena salud. Procuran un adecuado funcionamiento del hogar, que es su responsabilidad fundamental y generan acciones tendientes a garantizar el mantener esas condiciones.

Las buenas prácticas cotidianas en salud, no son sólo percibidas a nivel familiar, sino que también se manifiesta preocupación por la higiene en la comunidad: tratamiento de basura, vías de evacuación de las aguas pluviales, mantener los espacios públicos como comercios, parques, salones comunales y otros en buenas condiciones de salud. Hay una participación y sensibilización por involucrar a los diferentes miembros de la comunidad, por capacitarlos y sensibilizarlos hacia prácticas saludables y por resolver estos problemas colectivamente. No obstante lo anterior, no se reconoce una participación activa en la definición de las políticas institucionales en salud.

En cuanto a los mitos y las percepciones del rol de la mujer, se enfatiza el hecho de que la misma no se puede enfermar, pues es ella la responsable de mantener el bienestar del hogar y esto implica que aún durante su enfermedad guarda la responsabilidad del bienestar familiar, pese a que los miembros de la familia colaboran en diversas tareas para mantener el buen funcionamiento del hogar. Estas mujeres despliegan una gran cantidad de actividades de coordinación y de consecución de recursos en tiempo y trabajo de familiares y amigos para garantizar le bienestar de los miembros del grupo familiar.

Como se señaló previamente, cuando la atención requiere el acceso al medio institucionalizado de los servicios, el comportamiento que se asume es el de paciente, dejando de lado su participación activa como sujeto de salud.

\section{AGRADECIMIENTO}

Por este medio deseo expresar mis más sincero agradecimiento a todas aquellos que de una y otra forma han contribuido al desarrollo exitoso de la investigación. Sobre todo a la Vicerrectoría de Investigación de la Universidad de Costa Rica, por el apoyo presupuestario. Al Comité de Investigación de la Escuela de Enfermería por el apoyo brindado. A las mujeres de Tirrases que participaron en el estudio, porque sin ellas no hubiese sido posible el logro de la investigación. A las personas de la Corporación Canadiense AUCE Scala, por el apoyo presupuestario para la presentación de la investigación en la ciudad de Montreal, Canadá.

A todos ellos y ellas, mil gracias, Dios les bendiga siempre.

\section{BIBLIOGRAFÍA}

- Badilla, Leda. (1994) Género y Salud. Programa mujer, salud y desarrollo. O.P.S. Costa Rica.

- Buendía, Leonor et al. (1993) Métodos de Investigación Psicopedagógica. Mc Graw Hill. Madrid.

- Breilh, Jaime. (1990) La mujer y la triple jornada. Cepom.

- Lagarde, Marcela. (1997) Claves feministas para el poderío y la autonomía de las mujeres. Puntos de encuentro.

- León, Magdalena. (1998) Poder y Empoderamiento de las mujeres. Editorial TM. Colombia.

- Monge, Rocío y otra. (2000) Salud Mental y Conceptos Psicosociales en la salud de la mujer, U.C.R.

- Martínez F. et al.. (1998) Salud Pública. Mc Graw Hill. Madrid.

- O.P.S. Atención primaria en salud. (1978) Editorial Ginelda. New York.

- O.P.S. Género, mujer y salud en las Américas. (1993) Publicación científica N 541 Washington D.C.

- O.P.S. Protocolo de Investigación. Violencia Intrafamiliar. (1998) Washington.

- Pérez, Dellanira. Autoestima. Centro de nuestras vidas. Colectivo de Mujeres. San José.

- Profamilias. Hablemos de Salud Sexual y Derechos Reproductivos. (1998) Boletín No 4. Bogotá.

- Ruiz. Consuelo et al. (1991) La salud de las mujeres en atención primaria de salud. Sanidad Pública $\mathrm{N}^{\circ} 4$. Madrid.

- Taylor S.J. y otro. (1984) Introducción a los métodos cualitativos de investigación. Paidós, Barcelona.

- UNFPA. La promoción de la salud. Nuevo enfoque secretaría de salud. Handures.

- U.C.R. Análisis de la situación integral de salud, sector 1, 2, 3, Distrito de Tirrases. Programa de Salud U.C.R. - C.C.S.S. (1999) Costa Rica. 